NOE: a neutrino experiment for the CERN-Gran Sasso Long Base line project

\author{
E. Scapparone for the NOE Collaboration \\ Laboratori Nazionali del Gran Sasso \\ S.S.17 km 18+910, 61070, Assergi (AQ), Italy
}

Talk at 5th International Workshop on Tau Lepton Physics (TAU98)

Santander, Spain, September 14 - 17, 1998

To be published in Nucl. Phys. B, Proc. Suppl

( writeups available at http://www.ifca.unican.es/tau98/index_writeups.html) 


\title{
NOE: a neutrino experiment for the CERN-Gran Sasso Long Base line project
}

\author{
E. Scapparone ${ }^{\mathrm{a}}$ for the NOE Collaboration* \\ aINFN, LNGS S.S.17 km 18+910, 61070, Assergi (AQ), Italy
}

The project of a large underground experiment (NOE) devoted to long baseline neutrino oscillation measurement is presented. The apparatus, consisting of TRD and calorimeter modules, has been optimized to be sensitive in the region of $\sin ^{2} 2 \theta$ and $\Delta m^{2}$ suggested by the atmospheric neutrino oscillation signal.

\section{Introduction}

The scientific goal of the NOE long baseline (LBL) experiment is the measurement of neutrino masses looking at $\nu_{\mu} \rightarrow \nu_{e}$ and $\nu_{\mu} \rightarrow \nu_{\tau}$ oscillations. The philosophy of NOE design is to have oscillation sensitivity by looking for the $\tau$ decay $\left(\nu_{\mu} \rightarrow \nu_{\tau}\right.$ oscillation) or for an electron excess $\left(\nu_{\mu} \rightarrow \nu_{e}\right.$ oscillation) and by measuring a deficit of muons in apparent NC/CC ratio.

The main experimental hint for the $\nu$ oscillation search in the region of low $\Delta m^{2}\left(10^{-2} \div\right.$ $10^{-3} \mathrm{eV}^{2}$ ) comes from the muon deficit observed in atmospheric neutrino flux measurements [6 8]. Recent results from LBL reactor neutrino experiment (CHOOZ) excluded neutrino oscillation in $\bar{\nu}_{e}$ disappearance mode, up to $\sin ^{2} 2 \theta>0.18$ for large $\Delta m^{2}$ [9].

Taking into account the confirmations of the atmospheric neutrino anomaly and the negative CHOOZ result, a LBL experiment has to fulfill the following requirements:

1. $\nu_{\tau}$ tagging. The search for $\nu_{\tau}$ appearance is mandatory to confirm the oscillation phenomenon. This search requires detector high performances to tag the $\tau$ decay.

2. Measurement of the ratio NC/CC. This robust and unambiguos test is important to investigate on the existence of a neutrino oscillation signal. There is no doubt that this measurement can be done only with a massive detector.

*see [5] for the complete list of the Collaboration
3. Atmospheric neutrinos. After the last results from Superkamiokande, suggesting smaller values of $\Delta m^{2}$, the interest for the atmospheric neutrinos is raised up. It would be interesting to test this effect using a massive apparatus based on a different technique with respect to the water Čerenkov detectors.

4. Fast response. If a beam from CERN to Gran Sasso will be available in the next years, a strong competition with American and Japanese LBL programs is foreseen: at present, the 7 kton NOE project can adequately compete with the 8 kton MINOS detector and with K2K.

According to these remarks the NOE program can be summarized in this way:

- Direct $\nu_{\tau}$ appearance by kinematical $\tau$ decay reconstruction and inclusive $(\mathrm{NC} / \mathrm{CC})$ $\nu_{\mu}$ disappearance.

- Investigation of $\nu_{\mu} \rightarrow \nu_{e}$ oscillation in a mixing angle region two orders of magnitude beyond the CHOOZ limit.

- Atmospheric neutrino studies.

In order to improve the $\nu_{\tau}$ search, the apparatus has been equipped with Transition Radiation Detector (TRD) interleaved between calorimetric modules (CAL). The combination of TRD and CAL information strongly enforces $e, \mu$, and $\pi$ identification, thus permitting the study of the 
$\tau$ decay. In particular the $\tau$ appearence can be detected through the $\tau \rightarrow e \nu \nu$ channel, with a clean signature, taking advantage of the low background (residual $\nu_{e}$ beam). Moreover the good electron identification in the TRD and the low $\pi^{o}$ background allow to reach high $\sin ^{2} 2 \theta$ sensitivity looking for $\nu_{\mu} \rightarrow \nu_{e}$ oscillation, thus considerably enlarging the region investigated by CHOOZ.

\section{The NOE detector}

The detector (Fig. 1) is a typical fixed target apparatus consisting of a sequence of 12 basic modules(BM). Each BM is composed by a lighter part (TRD target) in which vertex, eidentification and kinematics are defined, followed by a scintillating fiber calorimeter devoted to energy measurement and event containement. The Basic Module (BM) is shown in Fig. 2. Appearance measurement is performed using events generated in TRD (2.4 kton), while disappearance measurements are performed by looking at events generated both in the TRD and in the calorimeter (total mass $\sim 7$ kton).

The calorimetric element is an 8 meter long bar filled with iron ore and scintillating fibers?. The calorimetric module is made by alternate planes of crossed bars. The calorimetric bar consists of more logical cells with square cross section. The iron ore is radiopure and practically cost free.

A lot of $R \& D$ has been performed to improve the scintillating fibers performance. The present production of $2 \mathrm{~mm}$ diameter scintillating fibers provide an attenuation length $\lambda=4.5 \mathrm{~m}$ and an light yield $\mathrm{L} \geq 20 \mathrm{pe} / \mathrm{MeV}$. These figures allow to build $8 m$ long bars. Further investigations to improve fiber features are in progress: longer fibers would allow to increase the NOE cross section $\left(9 \times 9 \mathrm{~m}^{2}\right)$ and therefore the total mass $(8 \mathrm{kton})$. It is worth noting the very high intrinsic granularity of the proposed calorimeter : the average distance between the fibers inside the absorber is of the order of $3 \mathrm{~mm}$. The fibers are grouped together at each side of the calorimetric bar and sent to single or multipixel photodetector.

The energy resolutions for electrons and

\footnotetext{
${ }^{2}$ Extruded scintillator strips with wavelength shifter fibers
} have been also studied.
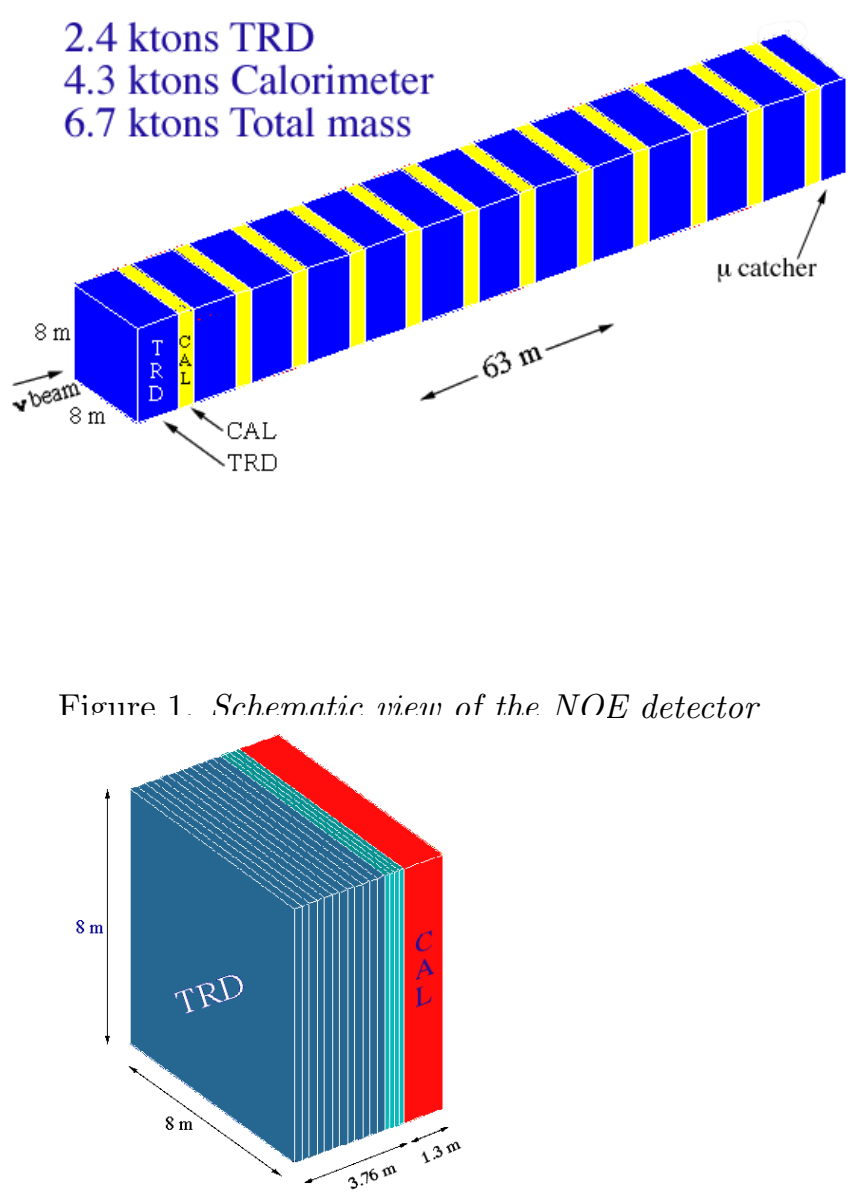

Figure 2. The Basic Module of the NOE detector

hadrons in the calorimeter have been evaluated by means of a GEANT Montecarlo simulation. They are, respectively, $\sigma(E) / E=0.01+0.17 / \sqrt{E}$ and $\sigma(E) / E=0.08+0.42 / \sqrt{E}$. The TRD module consists of 32 vertical layers of $8 \times 8 \mathrm{~m}^{2}$ area, each made by polyethylene foam radiator $\left(\rho \sim 100 \mathrm{mg} / \mathrm{cm}^{3}\right)$ and 256 proportional tubes $\left(3 \times 3 \mathrm{~cm}^{2}\right.$ cross section), filled with an $\mathrm{Ar}(60 \%)$ - $\mathrm{Xe}(30 \%)-\mathrm{CO}_{2}(10 \%)$ mixture, already tested in MACRO experiment. Consecutive layers have tubes rotated of $90^{\circ}$.

A graphite wall of $5 \mathrm{~cm}$ thickness is set in front 
of each of the first 24 layers of the TRD module acting as a 174 ton target for $\nu_{e}$ and $\nu_{\tau}$ interactions, to be identified in the following layers. The last target wall is followed by 8 TRD layers in order to identify the secondary particles. Each target wall corresponds to $0.25 X_{0}$ while the entire TRD basic module corresponds to about $7 X_{0}$ and $3.5 \lambda_{I}$. The total length is about $3.76 \mathrm{~m}$.

So many layers of proportional tubes permit to determine the muon energy by means of multiple measurements of energy loss $d E / d x$. Combining the informations coming from both subdetectors (TRD and CAL) the discrimination between $e$, $\mu$ and $\pi$ is largely enforced allowing the study of several neutrino oscillation channels.

\section{3. $\nu_{\tau}$ appearance and requirements about $\nu$ beam}

The rate of $\nu_{\tau} \mathrm{CC}$ events is given by

$R_{\tau}=A \int \sigma_{\tau} P_{o s c} \Phi d E$,

where $\mathrm{E}$ is the energy, $\sigma_{\tau}$ the $\nu_{\tau} \mathrm{CC}$ cross section, $P_{\text {osc }}$ the oscillation probability, $\Phi$ the muon neutrino flux and $A$ the number of target nucleons in the detector. The search for $\nu_{\tau}$ requires that the term $\sigma_{\tau} P_{o s c} \Phi$ is large. Therefore a dedicated $\nu$ beam has to provide most of its flux in the energy range where the factor $\sigma_{\tau} P_{\text {osc }}$ is larger.

Assuming the mixing of two neutrinos, the oscillation probability is

$P_{o s c}=\sin ^{2} 2 \theta \sin ^{2}\left(1.27 \Delta m^{2} L / E\right)$,

where $L=731 \mathrm{~km}$ is the distance CERN - Gran Sasso. We have to take into account that the $\tau$ cross section grows slowly with energy above a threshold of about $3.5 \mathrm{GeV}$. The factor $\sigma_{\tau} P_{o s c}$ is shown in Fig. 3 for different values of $\Delta m^{2}$. The optimal energy is about $15 \mathrm{GeV}$ for $\Delta m^{2}=$ $0.01 \mathrm{eV}^{2}$ and decreases gently with $\Delta m^{2}$ towards a limiting value of about $10 \mathrm{GeV}$.

In the following, the beam from Ref. [10] and 5 years of data taking are assumed.

\section{4. $\tau$ appearance searches}

Tau appearance search is performed on the basis of kinematical identification of the $\tau$ decay.
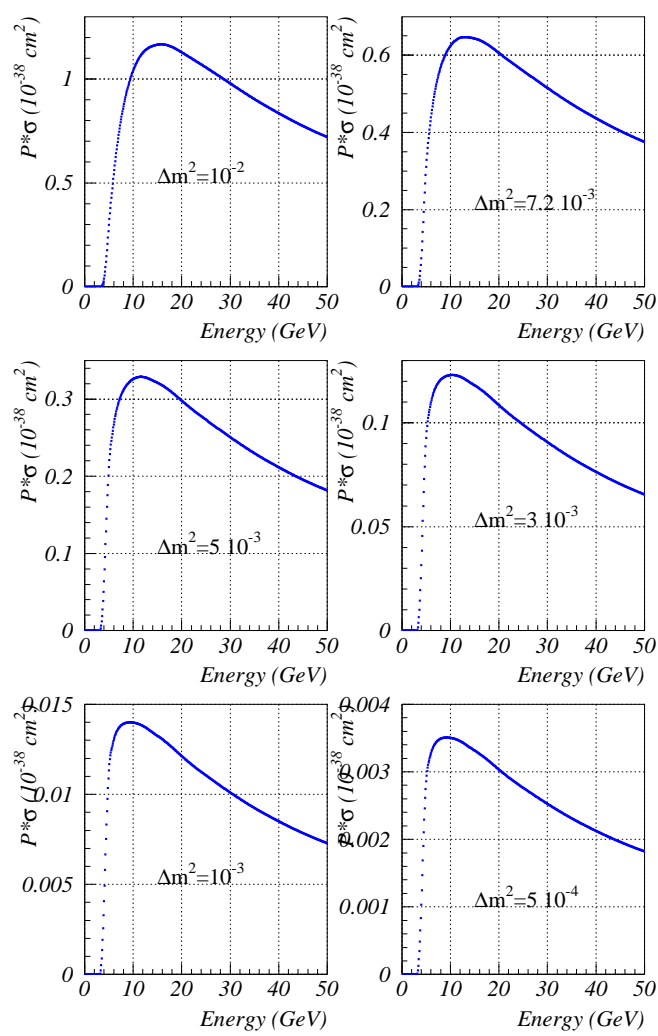

Figure 3. $\nu_{\tau} C C$ cross section multiplied by the oscillation probability for different values of $\Delta m^{2}$.

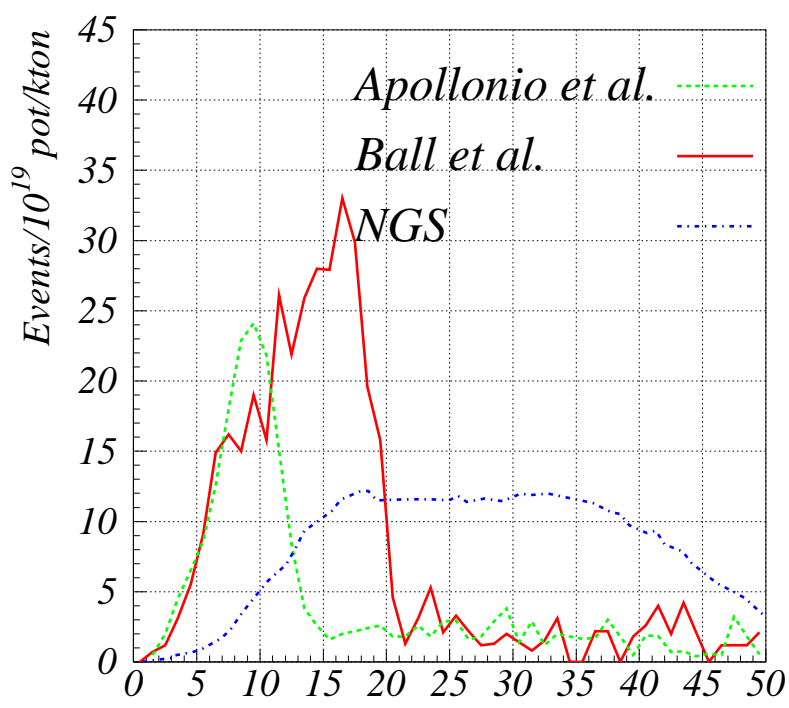

Figure 4. it $\nu_{\mu} \mathrm{CC}$ interaction spectra for different proposed beams. 
The $\tau \rightarrow e \nu \nu$ is the favourite channel for this search due to the low background level and the the good electron identification capability of the TRD. It is worth noting that in the region of atmospheric anomaly the oscillation probability is $50 \div 100$ times higher than expected in NOMAD, as a consequence a much lower background rejection power is required.

In order to check the overall NOE performances, a complete chain of event simulation and analysis has been performed. Event generators that include Fermi motion, $\tau$ polarization and nuclear rescattering inside the nucleus have been used to simulate quasi elastic, resonance and deep inelastic events.

Generated events are processed by a GEANT based MonteCarlo in which calorimeter and TRD geometrical set-up are described in detail, down to a scale of a few $\mathrm{mm}$. Fiber attenuation length, Birks saturation, photoelectron fluctuations and readout electronics non linearities for both TRD and calorimeter have been taken into account. DST of processed events $\left(\tau \rightarrow e \nu \nu, \nu_{\mu} \mathrm{NC}\right.$ and $\nu_{e}$ CC) have been produced and analysed.

Electron identification is performed by looking for high energy releases in the TRD and in the calorimeter readout elements in fully contained events. The electron candidate is the one that maximizes collected energy in a $5^{\circ}$ cone centered at the interaction vertex. Electron direction is reconstructed by weighting hits position by collected energy. With present algorithms an angular resolution of $0.6^{\circ}$ and a $180 \mathrm{MeV} / \mathrm{c}$ resolution on the measurement of transverse momentum are achieved.

The remaining part of the event is used to reconstruct the hadronic component. The obtained resolution on the measurement of transverse momentum is $420 \mathrm{MeV} / \mathrm{c}$.

Topological cuts on the electromagnetic shower are applied to reject $\nu_{\mu} \mathrm{NC}$ events with $\pi^{o}$ faking electrons. Fig. 6 shows a tipical neutral current event with a $\pi^{\circ}$ : the electromagnetic shower doesn't start in the event vertex, allowing an easy rejection of the event. Work is in progress to improve the reconstruction efficiency and $\nu_{\mu} \mathrm{NC}$ rejection. Additional cuts are performed to reduce the background:
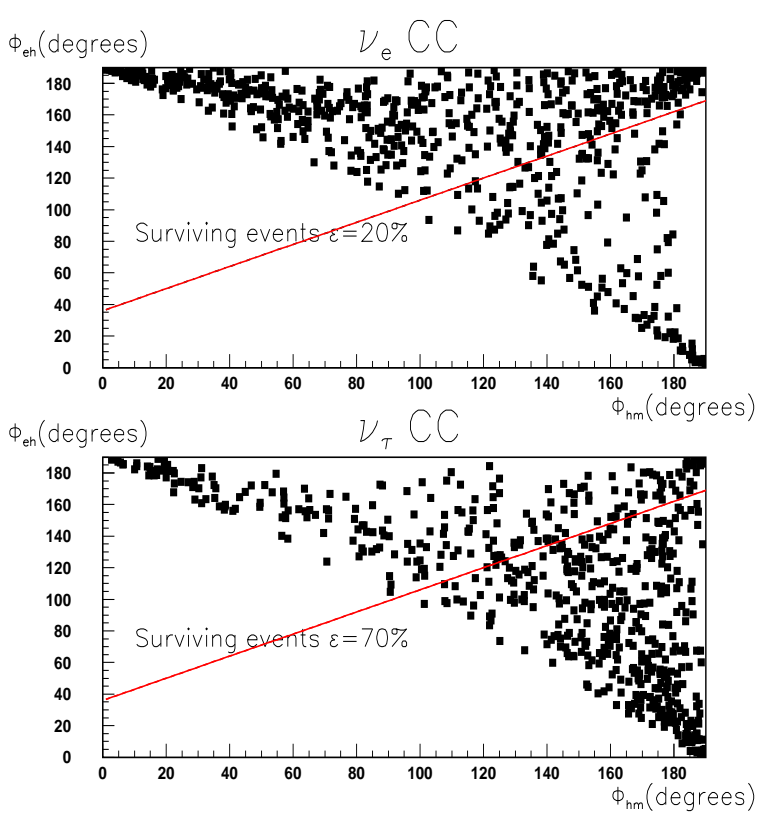

Figure 5. Effects of $\phi_{e-h} \phi_{m-h}$ cut on background $\left(\nu_{e}\right)$ and signal $\left(\nu_{\tau}\right)$.

- total reconstructed energy $<15 \mathrm{GeV}$,

- electron energy $>1.5 \mathrm{GeV}$,

- the component of electron momentum perpendicular to the hadronic jet direction $Q_{\text {lep }}>0.75 \mathrm{GeV} / \mathrm{c}$,

- transverse mass

$$
M_{T}=\sqrt{4 p_{T}^{e} p_{T}^{m} \sin ^{2}\left(\phi_{e-m} / 2\right)}<2 \mathrm{GeV},
$$

- $\phi_{e-h} \phi_{m-h}$ correlation as shown in Fig. 5.

Resulting efficiencies and residual backgrounds are reported in Table 1 .

\section{Neural network for the ratio NC/CC}

The measurement of the $\mathrm{NC} / \mathrm{CC}$ ratio is performed looking at the observable $R_{o b s}=($ no $\mu) / \mu$, where (no $\mu$ ) is the number of events not showing a muon track while $\mu$ is the number of events with a reconstructed muon. The procedure to perform 


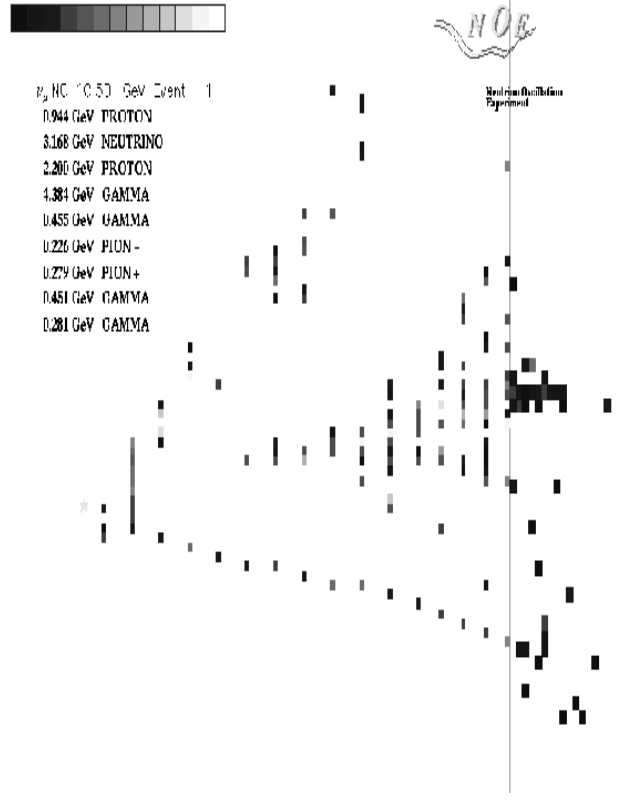

Figure 6. The event display of a typical NC event with $a \pi^{o}$. The hits on the left of the vertical line belong to the TRD, those on the right belong to the Calorimeter.

this measurement is well known [5]. The oscillation probability can be written as:

$P=\frac{R_{o b s}-R_{t h}-\epsilon\left(R_{t h}+1\right)}{R_{o b s}(1-\eta B)+\eta(1-B)}$

where $\epsilon$ is the ratio of $\nu_{e}$ to $\nu_{\mu}$ in the beam, $\mathrm{B}$ is the branching ratio for $\tau$ decay in muon, $R_{t h}=\sigma_{N C} / \sigma_{C C}$ and $\eta=\sigma_{\tau} / \sigma_{\mu}$. The last ratio has to be carefully evaluated. For istance, considering the NGS beam and $\Delta m^{2} \simeq 10^{-2}-10^{-3} \mathrm{eV}$, $\eta$ ranges between 0.25 and 0.32 . In this measurement, the systematics associated with $R_{t h}$, play of course an important role and can be in principle reduced by using a near detector. Nevertheless, near and far beams are not identical, introducing a possible systematic error, requiring a detailed study. From an experimental point of view the separation of (no $\mu$ ) events from $\mu$ events is usually supposed to be straightforward. On the contrary, this measurements become dif-

\begin{tabular}{|c|c|c|c|}
\hline Cut & Res. NC & Res. $v_{\mathrm{e}}$ beam & eff. $\tau \rightarrow \mathbf{e} v v$ \\
\hline 1 & $79 \%$ & $67 \%$ & $79 \%$ \\
\hline \multicolumn{4}{|c|}{ high released energy track in two views: $\mathbf{E y}=\mathbf{E z}$} \\
\hline Cut & Res. NC & Res. $v_{\mathrm{e}}$ beam & eff. $\tau \rightarrow \mathbf{e} v \nu$ \\
\hline 2 & $19 \%$ & $88 \%$ & $91 \%$ \\
\hline \multicolumn{4}{|c|}{$\begin{array}{l}\text { compact shower, starting from the vertex, topology } \\
\left(\pi^{0} \text { without initial conversion) }\right.\end{array}$} \\
\hline Cut & Res. NC & Res. $v_{\mathrm{e}}$ beam & eff. $\tau \rightarrow \mathbf{e} v v$ \\
\hline 3 & $11 \%$ & $88 \%$ & $89 \%$ \\
\hline \multicolumn{4}{|c|}{$\mathrm{E}_{\mathrm{Tot}}<15 \mathrm{GeV}$} \\
\hline Cut & Res. NC & Res. $v_{\mathrm{e}}$ beam & eff. $\tau \rightarrow \mathbf{e} v v$ \\
\hline 4 & $94 \%$ & $45 \%$ & $97 \%$ \\
\hline \multicolumn{4}{|c|}{$\mathrm{E}_{\text {e.m. }} \geq 1.5 \mathrm{GeV}$} \\
\hline Cut & Res. NC & Res. $v_{\mathrm{e}}$ beam & eff. $\tau \rightarrow \mathbf{e} v \nu$ \\
\hline 5 & $55 \%$ & $90 \%$ & $84 \%$ \\
\hline \multicolumn{4}{|c|}{$\mathrm{Q}_{\text {Lep }} \geq 0.75 \mathrm{GeV} / \mathrm{c}$} \\
\hline Cut & Res. NC & Res. $v_{\mathrm{e}}$ beam & eff. $\tau \rightarrow \mathbf{e} v v$ \\
\hline 6 & $25 \%$ & $75 \%$ & $70 \%$ \\
\hline \multicolumn{4}{|c|}{$\mathrm{M}_{\mathrm{T} \tau}<2 \mathrm{GeV}$} \\
\hline Cut & Res. NC & Res. $_{\mathrm{e}}$ beam & eff. $\tau \rightarrow \mathbf{e} v v$ \\
\hline 7 & $57 \%$ & $89 \%$ & $87 \%$ \\
\hline \multicolumn{4}{|c|}{$\phi_{\mathrm{e}, \mathrm{h}} \phi_{\mathrm{ptm}, \mathrm{h}}$ correlation } \\
\hline Cut & Res. NC & Res. $v_{\mathrm{e}}$ beam & eff. $\tau \rightarrow \mathbf{e} v v$ \\
\hline 8 & $35 \%$ & $20 \%$ & $70 \%$ \\
\hline
\end{tabular}

for $1.5 \times 10^{20}$ pot and $14000 v_{\mu} \mathrm{CC}$ (TRD) 5 years :

$\Delta \mathrm{m}^{2}=5 \times 10^{-3} \mathrm{eV}^{2} \quad 2.7 v_{\mathrm{e}} \mathrm{CC}+1.6 \mathrm{NC}=4.3 \mathrm{evs} . \quad 19 \tau$ evs. $\Delta \mathrm{m}^{2}=3 \times 10^{-3} \mathrm{eV}^{2} \quad 2.7 v_{\mathrm{e}} \mathrm{CC}+1.9 \mathrm{NC}=4.6$ evs. $7 \tau$ evs. no oscillation $\quad 2.7 v_{\mathrm{e}} \mathrm{CC}+2.0 \mathrm{NC}=4.7$ evs. $\quad 0 \tau$ evs.

Table 1

Details of analysis cuts, efficiency and rejection power for main background sources.

ficult at low energies, having the muons a shorter path length. In NOE analysis the identification of charged and neutral current events has been improved by means of a neural network. The algorithm uses 24 topological, geometrical and calorimetric event parameters as input. The network has been trained with $\nu_{\mu} \mathrm{CC}$ and NC MonteCarlo events with a energy uniformly distributed in the range $0 \div 50 \mathrm{GeV}$. The results of this analysis are shown in Fig. 7 . The $\mu$ event recognition is mainly dependent on the track length. For neutrino energy $E_{\nu} \geq 5 \mathrm{GeV}$ a good selection/rejection efficiency is obtained. As expected, the efficiency decreases with the energy, becoming the muon track shorter. 

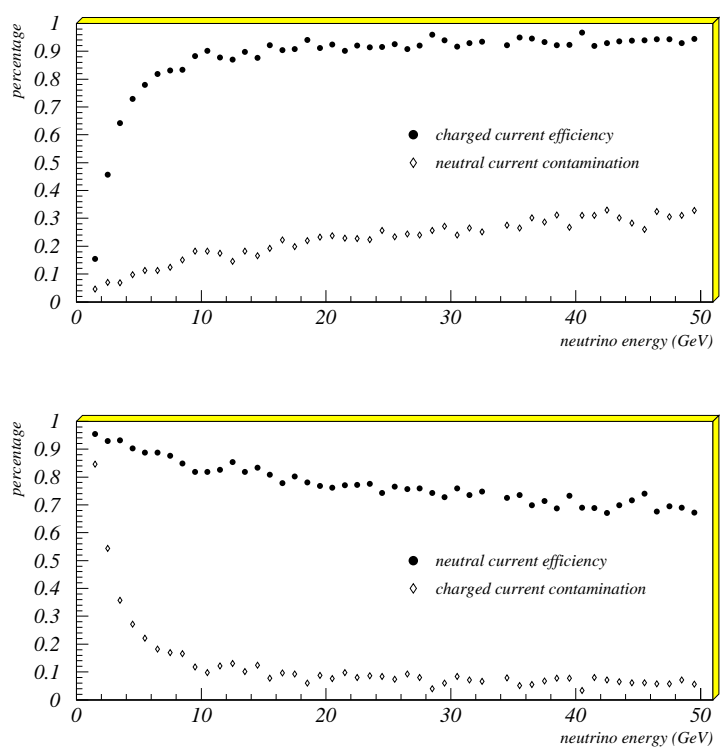

Figure 7. CC and NC signal. The contamination is also shown.

We are presently working to improve $\mathrm{NC}$ and $\mathrm{CC}$ separation at low energy. This measurement ( Fig. 8) allows a search for $\nu_{\mu} \rightarrow \nu_{\tau}$ oscillation down to $\Delta m^{2}=1 \cdot 10^{-3} \mathrm{eV}^{2}$.

\section{Conclusions}

The combined use of two subdetectors (TRD and CAL) allows to search for $\tau$ appearance signal for events generated in the 2.4 kton TRD target where electron identification, vertex and kinematics reconstruction are performed at best. Nevertheless the whole 8 kton mass can be exploited for disappearance oscillation tests.

Such measurements can be carried out at the same time by using an appropriate neutrino beam. A full analysis has recently been performed to demonstrate the feasibility of both measurements. In Fig. 8 the sensitivity of NOE experiment to $\nu$ oscillations is shown.

\section{REFERENCES}

1. G. Barbarino et al., INFN/AE-96/11
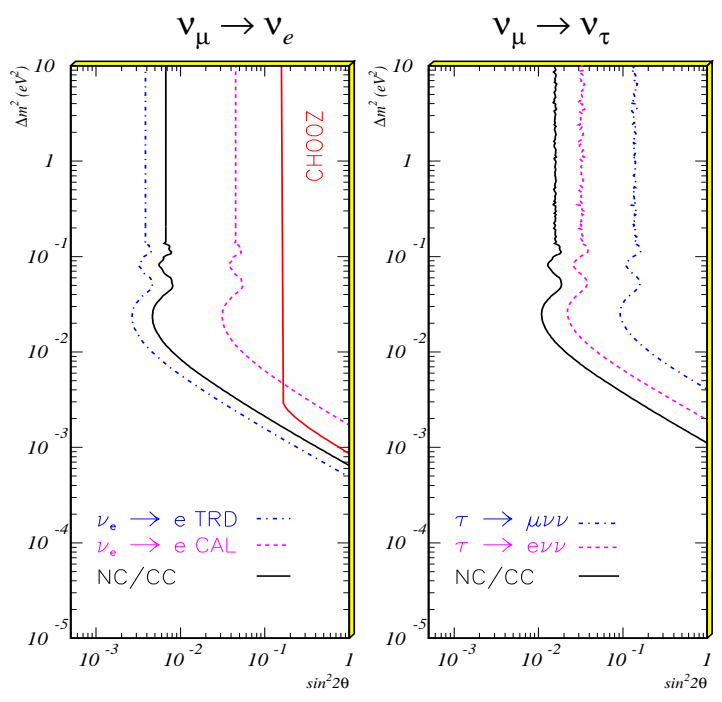

Figure 8. NOE sensitivity to $\nu$ oscillations.

2. G. Barbarino et al., Nucl. Phys. B (Proc. Suppl.) 48 (1996) 204

3. G. Osteria et al., Proceedings of the 7th Pisa Meeting on Advanced Detectors, Isola d' Elba, (1997)

4. G. Barbarino et al., Proceedings of TAUP97, Gran Sasso Laboratory, (1997) 223

5. G. Barbarino et al., INFN/AE-98/09

6. W.W.M. Allison et al. (Soudan Collaboration), Phys. Lett. B 391 (1997) 491

7. M. Ambrosio et al. (MACRO Collaboration), hep-ex/9807005

8. Y. Fukuda et al. (SuperKamiokande Collaboration), hep-ex/9807003

9. Chooz Collaboration, Phys. Lett. B $\mathbf{4 2 0}$ (1998) 397

10. A.E. Ball, S. Katsanevas and V. Vassilopouls, "Design Studies for a Long Baseline Neutrino beam" CERN/ECP 95-13

11. K. Elsener et al. "The CERN neutrino beam to Gran Sasso (NGS) - Conceptual technical design", CERN 98/02 and INFN/AE-98/05

12. M. Apollonio et al., Addendum to the Expression of Interest to the Gran Sasso Scientific Committee "Studies for a long baseline accelerator and atmospheric neutrino oscillation experiment at Gran Sasso" (1998) 